\title{
Effects of nano tantalum implants on inducing osteoblast proliferation and differentiation
}

\author{
XINYU LIU ${ }^{1-3}$, XIAOBIN SONG ${ }^{3}$, PENG ZHANG $^{4}$, ZHENKUN ZHU $^{1,2}$ and XIN XU ${ }^{1,2}$ \\ ${ }^{1}$ Stomatological College of Shandong University; ${ }^{2}$ Shandong Province Key Laboratory of Oral Biology Medicine; \\ ${ }^{3}$ Department of Stomatology, Qilu Hospital of Shandong University; ${ }^{4}$ Department of Orthopedics, \\ The Affiliated Hospital of Shandong University of Traditional Chinese Medicine, Jinan, Shandong 250012, P.R. China
}

Received July 1, 2016; Accepted September 20, 2016

DOI: $10.3892 /$ etm.2016.3801

\begin{abstract}
In the present study, we examined the effects of nano tantalum (Ta) dental implants on inducing osteoblast proliferation and differentiation. The MG-63 osteoblasts were divided into 3 groups after recovery, passage and storage: i) Osteoblast culturing group (control group); ii) osteoblast and titanium (Ti) implant co-culturing group (Ti group); and iii) osteoblast and Ta implant co-culturing group (Ta group). After 7 days, a scanning electron microscope was used to observe the growth status, number and morphological changes of the cells on the surfaces of the materials. An MTT assay was used to detect cell proliferation after culturing for 1, 3 and 7 days. ELISA assay was used to detect the levels of alkaline phosphatase (ALP) after 1, 3 and 7 days. Western blot analysis was used to detect the expression levels of collagen type I (Col-1) and osteocalcin after 1, 3 and 7 days. There was significant cell spreading on the surfaces of Ti and of Ta after 7 days, flat and with many pseudopodia. Additionally, there were more cell components in the Ta group. Concurrently, cell proliferation in the Ti and Ta groups increased. There was also an increase in the level of ALP and the expression level of Col-1 over time. The indexes of the Ta group were more apparent than those of the Ti group at each time-point, and the differences were statistically significant $(\mathrm{p}<0.05)$. In conclusion, compared with Ti implants, Ta implants induced more osteoblast proliferation and differentiation.
\end{abstract}

\section{Introduction}

Dental implants have become one of the most effective ways to treat dental defects and repair dental system function (1). The

Correspondence to: $\mathrm{Dr} \mathrm{Xin} \mathrm{Xu}$, Stomatological College of Shandong University, 44-1 Wenhua West Road, Jinan, Shandong 250012, P.R. China

E-mail: xu_xin1212@163.com

Key words: nano tantalum implants, osteoblast, proliferation, differentiation, alkaline phosphatase, collagen type I, osteocalcin key to success of dental implants and restoration is forming a stable osseointegration between implants and bone interfaces, namely, reliable physical and chemical bonding (2).

Titanium (Ti), with its excellent mechanical properties and biocompatibility, is the most widely used implant material, but it is biologically inert (3). After implantation, peripheral tissues show a non-specific reaction and a random property (4). It needs a long healing period to form a functional restoration. As a new restoration material, nano tantalum (Ta) has been confirmed as optimal for the proliferation, differentiation and mineralization of osteoblasts (5). Nano structure is better for the growth of the osteoblasts than a micron structure (6). By analyzing the effects of Ta implants on inducing osteoblast proliferation and differentiation, the current study lays a theoretical foundation for clinical application.

\section{Materials and methods}

Cell recovery, passage and preservation. Supplies included MG-63 osteoblasts (Cell Bank of Chinese Academy of Science, Shanghai, China), 0.25\% trypsin (Gibco Services, Inc., Amarillo, TX, USA) and Dulbecco's modified Eagle's medium (DMEM) culture medium containing double-antibody, $10 \%$ calf serum and $\mathrm{NaHCO}_{3}$ (Gibco Services, Inc.). We also used micropipettes (Gilson, Inc., Villiers-le-Bel, France), an HB2460 super-clean bench, Micro Centaur desk centrifuge (MSE Limited, London, England) and a phase contrast microscope (Nikon, Tokyo, Japan). The cells were processed with conventional recovery, passage and preservation.

Experimental grouping. Groups were divided into the osteoblast culture group (the control group), osteoblast and Ti implant co-culture group (Ti group), and osteoblast and Ta implant co-culture group (Ta group). A grade 4 commercially pure Ti sheet of 10x1.5 mm (Baoji Lihua Non-ferrous Metals Co., Ltd., Baoji, China) and a Ta sheet of 10x1.5 mm (Zimmer Biomet, Warsaw, IN, USA) were used for treatments. A pre-treatment and heat treatment of Ti and Ta sheets were performed. Anodic oxidation under $10 \mathrm{~V}$ for $2 \mathrm{~h}$ was conducted. A concentration of MG-63 osteoblasts was regulated to $2.5 \times 10^{4} / \mathrm{ml}$. A cell suspension of $1 \mathrm{ml}$ was added into separate wells and placed in a culture plate with 12 wells. This was then cultured with conventional fetal bovine serum 
Table I. Cell proliferation (OD value).

\begin{tabular}{lccccr}
\hline Groups & 1 day & 3 days & 7 days & F-value & P-value \\
\hline Control & $0.09 \pm 0.02$ & $0.10 \pm 0.03$ & $0.10 \pm 0.04$ & 0.532 & 0.685 \\
Ti & $0.25 \pm 0.06$ & $0.36 \pm 0.08$ & $0.42 \pm 0.10$ & 5.324 & 0.036 \\
Ta & $0.33 \pm 0.09$ & $0.42 \pm 0.12$ & $0.48 \pm 0.13$ & 5.865 & 0.030 \\
F-value & 5.754 & 5.968 & 6.230 & & \\
P-value & 0.031 & 0.028 & 0.025 & & \\
\hline
\end{tabular}

OD, optical density; Ti, titanium; Ta, tantalum.

DMEM culture medium and incubated in a $\mathrm{CO}_{2}$ incubator. The culture medium was changed every 2 days.

Cell growth on the material surface. We used 25\% glutaric acid to fix samples washed with phosphate-buffered saline (PBS). These were then dehydrated with gradient alcohol and desiccated to a critical point before being sprayed on a vacuum surface. Growth, quantities and morphological changes of cells on the material surface were observed through a scanning electron microscope (SEM) (Olympus, Tokyo, Japan).

Cell proliferation at 1, 3 and 7 days tested by 3-(4,5-dimethylthiazolyl-2-yl)-2,5-diphenyltetrazolium bromide (MTT) method. We added MTT solution into the well of the 12-well plate $(5 \mathrm{mg} / \mathrm{ml})$ with $20 \mu \mathrm{l} /$ well. The samples were incubated at $37^{\circ} \mathrm{C}$ for $4 \mathrm{~h}$, followed by terminating the culture. The supernatant was discarded without destroying the blue crystal layer attached to the material surface. Dimethyl sulfoxide of $150 \mu \mathrm{l}$ was added into each well and placed at $25^{\circ} \mathrm{C}$. The material was oscillated for $20 \mathrm{~min}$ until the crystals were fully dissolved. The material was placed into a 96-well plate, with zero modulation in distilled water. A selected wavelength of $490 \mathrm{~nm}$ on an ELISA reader (PerkinElmer, Inc., Waltham, MA, USA) was used to determine the value of light absorption [optical density (OD) value]. We measured 5 wells/group and took the mean values of each one.

Alkaline phosphatase (ALP) level at 1, 3 and 7 days tested by ELISA method. We transferred samples of various groups into a new 24 -well plate, using $0.01 \mathrm{~mol} / \mathrm{l} \mathrm{PBS}$ to wash 3 times and drain. We then added $50 \mu 1$ Triton X-100 $(1 \mathrm{~g} / \mathrm{l})$ into each well, leaving it overnight at $4^{\circ} \mathrm{C}$ after repeated pipetting. A substrate in ALP kits was added at $100 \mu \mathrm{l} /$ well. The material was placed in an incubator for $30 \mathrm{~min}$ at $37^{\circ} \mathrm{C}$ before adding $0.2 \mathrm{~mol} / \mathrm{l} \mathrm{NaOH}$ of $50 \mu \mathrm{l}$ to terminate the reaction. A selected wavelength of A410 $\mathrm{nm}$ on an ELISA reader was used to test the OD value and take the mean value.

Expression level of type I collagen protein (Col-1) and osteocalcin at 1, 3 and 7 days tested by western blot analysis. We added RIPA lysate and PMSF liquid into cells and set the material on ice for $10 \mathrm{~min}$. Ultrasonic focalization decomposition was performed for $1 \mathrm{~min}$ at $4^{\circ} \mathrm{C}$ with $12,000 \mathrm{x}$ g of liquid centrifuge for $15 \mathrm{~min}$. A test concentration of protein through Bradford colorimetry was attained and preserved at $-20^{\circ} \mathrm{C}$ for inspection. We added 5X SDS loading buffer into each protein sample proportionally before heating for $5 \mathrm{~min}$ at $95^{\circ} \mathrm{C}$ and 12,000 x g centrifuging for $2 \mathrm{~min}$. After absorbing the supernatant and adding the gel-like substance to each well, we developed a regulated protein concentration of $20 \mu \mathrm{l} /$ well. We added $10 \mu \mathrm{l}$ pre-stained protein molecular weight marker into one of the wells and applied electrophoresis for $30 \mathrm{~min}$ under $40 \mathrm{~V}$ with a vertical protein electrophoresis apparatus (Bio-Rad Laboratories, Inc., Hercules, CA, USA). A separation gel was added when reaching a dye strip, and the voltage was changed to $60 \mathrm{~V}$. Electrophoresis was continued for 60 min under constant voltage until bromine phenol blue rose to the bottom of the gel. At this point, we turned off the power. Conventional transmembrane channels and the PVDF membrane were sealed. Rabbit polyclonal anti-Col-1 antibody (cat. no. sc-59772; dilution, 1:500), rabbit monoclonal osteocalcin antibody (cat. no. sc-376726; dilution, 1:500) and rabbit polyclonal $\beta$-actin (cat. no. sc-47778; dilution, 1:500) (all from Santa Cruz Biotechnology, Inc., Santa Cruz, CA, USA) were added into a hybrid bag. The material was oscillated and incubated overnight at $4^{\circ} \mathrm{C}$, then washed with PBS for 10 min x 5 times. A diluted secondary antibody labeled with HRP with blocking solution in proportion of 1:5,000 was added and oscillated for $1 \mathrm{~h}$ before being washed with PBS for 10 min x 5 times Pierce ECL kits (Thermo Fisher Scientific, Inc., Waltham, MA, USA) and Image-Pro Plus software (Media Cybernetics, Rockville, MD, USA) were used to develop, expose, photograph and analyze the material.

Statistical analysis. SPSS 20.0 (IBM SPSS, Armonk, NY, USA) was used for statistical analysis. Measurement data are expressed as mean \pm standard deviation, with comparisons among groups analyzed by a one-way ANOVA comparison through LSD between two groups. A comparison within groups was analyzed by variance of repeated measurement data. $p<0.05$ was considered to indicate a statistically significant difference.

\section{Results}

Cell growth on the material surface. Cells on the material surface of the control group were insignificant 7 days after culture. The cells bulged and were cord-like. Many cells stretched on the Ti and Ta sheet surfaces and presented a flat shape, with many pseudopodia. However, more cells were produced on the Ta sheet (Table I). 
Table II. Expression level of Col-1 and osteocalcin.

\begin{tabular}{|c|c|c|c|c|c|c|c|c|c|c|}
\hline \multirow[b]{2}{*}{ Groups } & \multicolumn{5}{|c|}{ Col-1 } & \multicolumn{5}{|c|}{ Osteocalcin } \\
\hline & 1 day & 3 days & 7 days & F-value & P-value & 1 day & 3 days & 7 days & F-value & P-value \\
\hline Control & $0.06 \pm 0.02$ & $0.05 \pm 0.02$ & $0.07 \pm 0.03$ & 0.234 & 0.852 & $0.04 \pm 0.02$ & $0.03 \pm 0.02$ & $0.05 \pm 0.02$ & 0.316 & 0.765 \\
\hline $\mathrm{Ti}$ & $0.25 \pm 0.06$ & $0.28 \pm 0.10$ & $0.32 \pm 0.12$ & 5.233 & 0.034 & $0.26 \pm 0.09$ & $0.30 \pm 0.12$ & $0.34 \pm 0.14$ & 5.326 & 0.032 \\
\hline $\mathrm{Ta}$ & $0.36 \pm 0.08$ & $0.40 \pm 0.12$ & $0.43 \pm 0.14$ & 5.754 & 0.030 & $0.35 \pm 0.07$ & $0.42 \pm 0.13$ & $0.46 \pm 0.15$ & 5.869 & 0.027 \\
\hline F-value & 7.521 & 7.123 & 7.627 & & & 6.569 & 6.857 & 7.105 & & \\
\hline P-value & 0.013 & 0.015 & 0.013 & & & 0.025 & 0.021 & 0.013 & & \\
\hline
\end{tabular}

Col-1, collagen type I; Ti, titanium; Ta, tantalum.

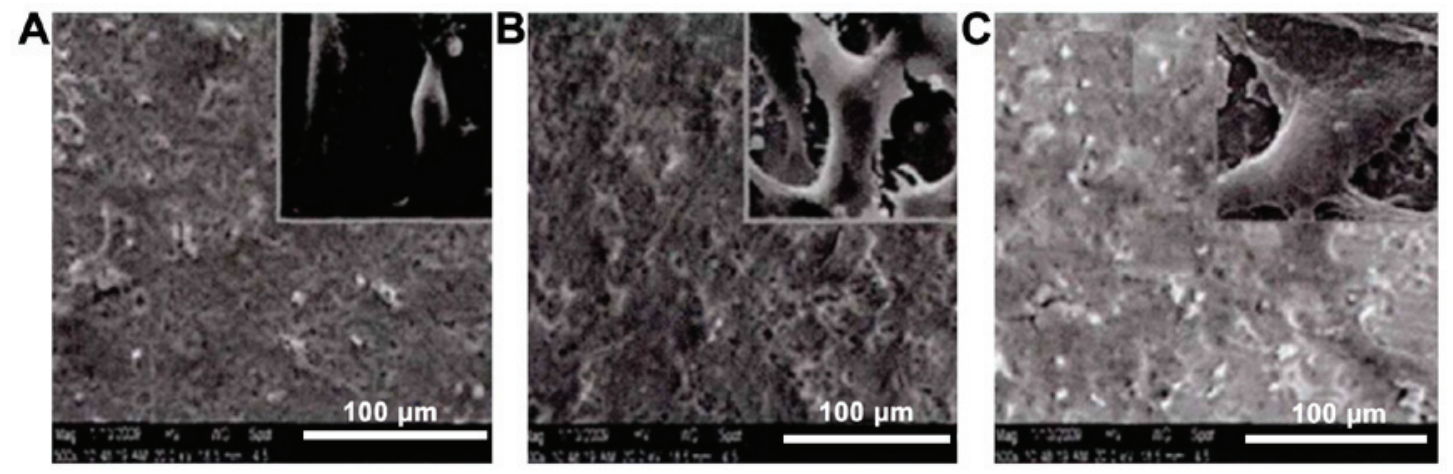

Figure 1. Cell growth on the material surface observed by SEM. (A) Control group; (B) Ti group; and (C) Ta group. SEM, scanning electron microscope; Ti, titanium; Ta, tantalum.

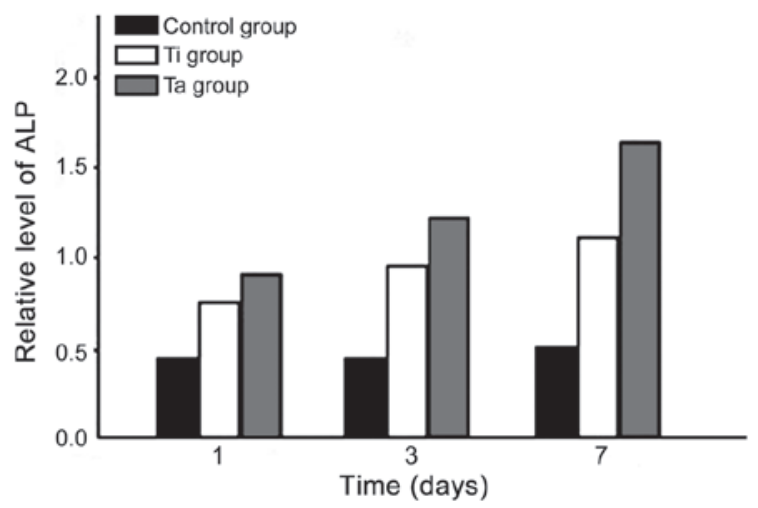

Figure 2. Comparison of ALP levels. ALP, alkaline phosphatase.

Cell proliferation. The proliferation of cells in the Ti and Ta groups increased as time progressed. Furthermore, the cell proliferation rate of the Ta group increased over time when compared with the Ti group. The difference was statistically significant $(\mathrm{p}<0.05)$ (Table I).

ALP level. The ALP levels of the Ti and Ta groups increased as time progressed. The level in the Ta group was higher at various times when compared with the Ti group. The difference was statistically significant ( $<<0.05)$ (Fig. 2).

Expression level of Col-1 and osteocalcin. The expression level of Col-1 and osteocalcin of the Ti and Ta groups increased over time. The level in the Ta group was higher at various times. The difference was statistically significant $(\mathrm{p}<0.05)$ (Table II).

\section{Discussion}

Osteoblasts are the most important functional cells in the bone remodeling process and are among the most active cells during the formation of the implant-bone interface. Maturely differentiated osteoblasts secrete collagen and non-collagenous proteins in bone matrix, and the cytoplasm is basophilic (7). Those secretions can be synthesized as membrane-bound ALP, which are related to mineralization (8). Basophilic cytoplasm can be released by phospholipid phosphatidylinositol-specific phospholipase $\mathrm{C}$, which may be related to transmembrane signal pathways (9). Osteocalcin is the main non-collagenous protein in the bone matrix and is now considered as a mark of the latest expression in the osteoblasts (10).

Findlay et al compared the effects of Ta, Ti, cobalt chrome and plastic products for culturing use on osteoblasts (11). On day 3 of our study, osteoblast proliferation in the plastic products was slightly faster than that in Ta. On day 7, the Ta group was superior to other material groups in terms of either the absolute number or division number of cells, but there was no difference in mRNA expression, osteoblast activity and mineralization extent. This suggested that Ta was a good substance for attachment, development and differentiation of osteoblasts. It also was suitable for the proliferation of osteoblasts, not significantly different from the plastic articles (which are 
usually taken as a BenchMark). Sakai et al tested the influence of 14 kinds of metals and a non-metallic substance on the in vitro viability of osteoblast-like cells (12). The study found that the influence of cells on particles depended on the direct action between particles and cells, indirect action of dissolved ions and the type of particle elements. The cytotoxicity of Ta particles $(<44 \mu \mathrm{m})$ and their extractives were low. When cells were co-cultured with Ta and other metal particles for 3 days, the cytotoxicity was lower than that of the control group. However, it was similar to the control group on day 6 , which illustrated that cells could be restored and proliferated after the first damage (13). Recent studies show that nanoparticles in the body can induce autophagy, a double-edged sword. Nanoparticles in normal cells could induce cytotoxicity, which should be avoided. Conversely, nanoparticles can be used in the treatment of disease in specific cells, such as for neurodegenerative diseases, including Parkinson's disease (14).

There was abundant cell spreading on the surfaces of the Ti and Ta sheets after 7 days, with flat cells and many pseudopodia. Additionally, there were more cell components in the Ta group. Concurrently, cell proliferation in the Ti and Ta groups increased. The level of ALP and the expression level of Col-1 and osteocalcin also increased over time. The indexes of the Ta group were more apparent than those of the Ti group at each time-point, and the differences were statistically significant $(\mathrm{p}<0.05)$. In conclusion, the nano $\mathrm{Ti}$ implants resulted in improved proliferation and differentiation of osteoblasts.

\section{Acknowledgements}

The present study was supported by the Scientific Research Project for Middle-aged and Young Scientists of Shandong Province (no. BS2013SW041) and the Natural Science Foundation of China (no. 81271105).

\section{References}

1. Mantripragada VP, Lecka-Czernik B, Ebraheim NA and Jayasuriya AC: An overview of recent advances in designing orthopedic and craniofacial implants. J Biomed Mater Res A 101: 3349-3364, 2013.
2. Velasco-Ortega E, Alfonso-Rodríguez CA, Monsalve-Guil L, España-López A, Jiménez-Guerra A, Garzón I, Alaminos M and Gil FJ: Relevant aspects in the surface properties in titanium dental implants for the cellular viability. Mater Sci Eng C 64: 1-10, 2016.

3. Hotchkiss KM, Ayad NB, Hyzy SL, Boyan BD and OlivaresNavarrete R: Dental implant surface chemistry and energy alter macrophage activation in vitro. Clin Oral Implants Res 3: 12-13, 2016.

4. Sollazzo V, Pezzetti F, Massari L, Palmieri A, Brunelli G, Zollino I, Lucchese A, Caruso G and Carinci F: Evaluation of gene expression in MG63 human osteoblastlike cells exposed to tantalum powder by microarray technology. Int J Periodontics Restorative Dent 31: e17-e28, 2011

5. Chang YY, Huang HL, Chen YC, Hsu JT, Shieh TM and Tsai MT: Biological characteristics of the MG-63 human osteosarcoma cells on composite tantalum carbide/amorphous carbon films. PLoS One 9: e95590, 2014.

6. Deng $J$ and Wang $Y$ : The metal tantalum in orthopedic applications. Sheng Wu Yi Xue Gong Cheng Xue Za Zhi 28: 419-422, 2011 (In Chinese).

7. Frandsen CJ, Brammer KS, Noh K, Johnston G and Jin S: Tantalum coating on $\mathrm{TiO}_{2}$ nanotubes induces superior rate of matrix mineralization and osteofunctionality in human osteoblasts. Mater Sci Eng C 37: 332-341, 2014.

8. Ninomiya JT, Struve JA, Krolikowski J, Hawkins M and Weihrauch D: Porous ongrowth surfaces alter osteoblast maturation and mineralization. J Biomed Mater Res A 103: 276-281, 2015.

9. Sagomonyants KB, Hakim-Zargar M, Jhaveri A, Aronow MS and Gronowicz G: Porous tantalum stimulates the proliferation and osteogenesis of osteoblasts from elderly female patients. J Orthop Res 29: 609-616, 2011.

10. Balla VK, Bodhak S, Bose S and Bandyopadhyay A: Porous tantalum structures for bone implants: fabrication, mechanical and in vitro biological properties. Acta Biomater 6: 3349-3359, 2010.

11. Findlay DM, Welldon K, Atkins GJ, Howie DW, Zannettino AC and Bobyn D: The proliferation and phenotypic expression of human osteoblasts on tantalum metal. Biomaterials 25: 2215-2227, 2004.

12. Sakai T, Takeda S and Nakamura M: The effects of particulate metals on cell viability of osteoblast-like cells in vitro. Dent Mater J 21: 133-146, 2002.

13. Ha SW, Weitzmann MN and Beck GR Jr: Bioactive silica nanoparticles promote osteoblast differentiation through stimulation of autophagy and direct association with LC3 and p62. ACS Nano 8: 5898-5910, 2014.

14. Zhao Y, Howe JLC, Yu Z, Leong DT, Chu JJ, Loo JS and Ng KW: Exposure to titanium dioxide nanoparticles induces autophagy in primary human keratinocytes. Small 9: 387-392, 2013. 